\title{
Colugos: obscure mammals glide into the evolutionary limelight Robert D Martin
}

Address: Department of Anthropology, The Field Museum, Chicago, IL 60605-2496, USA. Email: rdmartin@fieldmuseum.org

Published: I May 2008

Journal of Biology 2008, 7:13 (doi:10.1186/jbiol74)

The electronic version of this article is the complete one and can be found online at http://jbiol.com/content/7/4/13

(C) 2008 BioMed Central Ltd

\begin{abstract}
Substantial molecular evidence indicates that tree-shrews, colugos and primates cluster together on the mammalian phylogenetic tree. Previously, a sister-group relationship between colugos and primates seemed likely. A new study of colugo chromosomes indicates instead an affinity between colugos and tree-shrews.
\end{abstract}

Colugos, constituting the obscure and tiny order Dermoptera, are gliding mammals confined to evergreen tropical rainforests of South-East Asia. There are two extant species, now placed in separate genera: Galeopterus variegatus (Malayan colugo, formerly known as Cynocephalus variegatus) and Cynocephalus volans (Philippine colugo). Their most obvious hallmark is a gliding membrane (patagium) surrounding almost the entire body margin. Colugos are also called 'flying lemurs', but - as Simpson aptly noted [1] they "are not lemurs and cannot fly". They differ from other gliding mammals (certain rodents and marsupials) in that the patagium also extends between the hind limbs and the short tail, even stretching between fingers and toes (hence the name 'mitten-gliders'). The lower incisors are unique: the forward-leaning (procumbent) crown of each tooth is subdivided into several comb-like tines. Colugos are strict herbivores, predominantly eating young leaves from many tree species, and in the gastrointestinal tract the caecum is greatly enlarged. Their habits are poorly documented, although a recent field study yielded valuable new information [2].

Colugos would doubtless still be languishing in obscurity but for mounting evidence indicating a connection with primate evolution. In fact, Gregory [3] presaged this long ago by proposing the superorder Archonta for elephant- shrews, tree-shrews, colugos, bats and primates. However, Simpson's ensuing influential classification of mammals [1] rejected this assemblage. Subsequently, prompted by Butler [4], the superorder Archonta was progressively resuscitated, although most authors emphatically excluded elephantshrews (for example [5,6]). A quite recent major classification of mammals [7] united tree-shrews, colugos, bats and primates in the grand order Archonta.

This whole topic has been reinvigorated by molecular evidence indicating that tree-shrews, colugos and primates, at least, may be quite closely related. Despite general agreement over the clustering of tree-shrews, colugos and primates together, the placement of colugos within this group in relation to primate evolution is still a matter of debate. Some lines of evidence place tree-shrews and colugos together in a sister group to primates, whereas other workers in the field have advocated that colugos have a closer affinity to primates than to tree-shrews. This debate is nicely highlighted by a recent paper by Nie et al. [8] in $B M C$ Biology, which provides cytogenetic evidence for the treeshrews and colugos as a sister group to primates. In contrast, Janecka et al. [9] in a recent paper last year come to the different conclusion that colugos form a sister group more closely related to primates than to tree-shrews on the basis of nuclear DNA sequence data. 


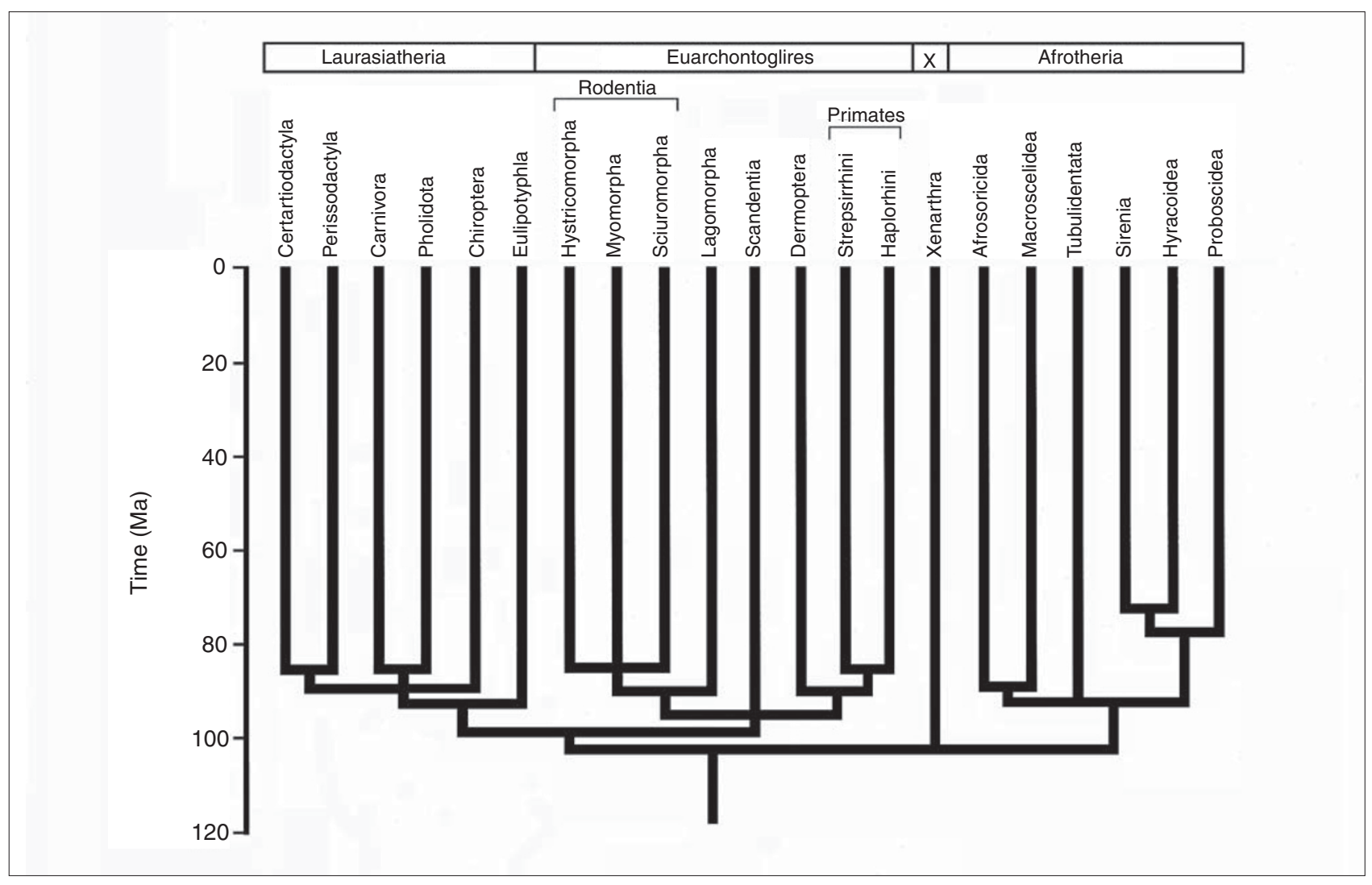

Figure I

Simplified tree showing relationships between 18 extant orders of placental mammals, inferred from a consensus phylogeny integrating molecular evidence [20]. Separate suborders are shown for Rodentia $(n=3)$ and Primates $(n=2)$. Four superorders have been recognized (top bar; $X=$ Xenarthra). Note the relatively rapid diversification of placental orders between 80 and 100 million years ago (Ma).

\section{The molecular revolution}

Determination of higher-level relationships among placental mammals using morphological evidence proved remarkably challenging [6,10-17]. Despite general agreement about subdividing placental mammals into orders, recognition of deeper nodes in the tree has been tentative at best (see for example [18]), and morphological interpretations, such as the placement of colugos with bats in the grouping Volitantia $[6,10,11]$, frequently clash with the molecular evidence. The rapidly accumulating molecular evidence has yielded an entirely new perspective on placental mammal evolution. Phylogenetic reconstructions using comprehensive DNA datasets (see for example [19]) have led to consistent recognition of four monophyletic superorders: Afrotheria, Euarchontoglires, Laurasiatheria and Xenarthra. Those superorders were confirmed by the most extensive analysis to date, generating a supertree combining results from more than 2,500 partial trees [20] (Figure 1). The superorder Euarchontoglires (alternatively known as Supraprimates [21-23]) is often divided into two subgroups:
Euarchonta (Dermoptera (colugos), Primates and Scandentia (tree-shrews)) and Glires (Lagomorpha (rabbits and hares) and Rodentia (rodents)). However, although the consensus supertree [20] portrays Euarchontoglires as monophyletic, internal relationships between tree-shrews, Glires and colugos+primates appear as an unresolved trichotomy (Figure 1). It should also be noted that tree-shrews and lagomorphs (usually linked to rodents in Glires) have emerged as sister groups in several individual studies: those of the $\varepsilon$-globin gene [24]; exon 28 of the von Willebrand factor gene [25]; complete protein-coding mitochondrial DNA sequences [26]; and complete sets of tRNA and rRNA sequences from mitochondrial genomes [27]. Figure 2 shows a version of this part of the tree with possible sister groups indicated.

The now widely recognized taxon Euarchonta is a radically pruned version of Gregory's Archonta, excluding not only elephant-shrews (now placed in Afrotheria) but also bats (Chiroptera, in Laurasiatheria). Strikingly, molecular evidence 
uniformly indicates a very deep separation between colugos and bats, soon after the common ancestor of extant placental mammals (see Figure 1). Similarities that led morphologists to recognize the Volitantia probably reflect convergent gliding adaptations in colugos and the (hitherto undocumented) precursors of bats.

Investigation of short interspersed nuclear elements (SINEs) in Euarchontoglires [22,23] identified Euarchonta and Glires as monophyletic sister taxa, but left the relationship between colugos, tree-shrews and primates within the Euarchonta as an unresolved trichotomy (see Figure 2). SINEs originate from retroposition of small RNAs as localized insertions throughout eukaryote genomes. Potentially, they are highly informative phylogenetic markers because retroposition at exactly the same site in independent lineages (that is, convergent evolution) is highly unlikely. SINEs derived from 7SL RNA seem to be a shared derived feature of Euarchontoglires, subsequently leading to dimeric Alu sequences in primates, chimeric sequences in treeshrews and B1 sequences in rodents [23]. Moreover, a genome search revealed five independent retroposon insertions shared by tree-shrew and human and fourteen shared by mouse, rat and rabbit, indicating a basal divergence between Euarchonta and Glires. But the very limited genomic information available for colugos and lagomorphs handicapped this study [23], such that relationships between colugos, primates and tree-shrews were left unresolved. Some reports $[28,29]$ based on mitochondrial DNA sequences challenged the monophyly of primates, linking colugos to higher primates (Anthropoidea) to the exclusion of prosimians (lemurs, lorisiforms and tarsiers). Schmitz et al. [30] replicated this aberrant finding, and conducted a test using SINEs. They identified a substantial set of transposable elements present in all major groups of extant primates but lacking in colugo, thus clearly supporting primate monophyly.

\section{An eternal triangle}

Within the Archonta, colugos have sometimes been linked most closely to tree-shrews and sometimes to primates. Support for the former association has now been provided by Nie et al. [8], who generated a G-banded karyotype for the Malayan colugo G. variegatus and used reciprocal chromosome painting with human and $G$. variegatus chromosome-specific probes to establish the first genomewide comparative map matching Galeopterus to human. This enabled them to define 44 segments in the G. variegatus genome homologous to segments in humans. Comparisons across similar published maps from other species within Euarchontoglires revealed that Galeopterus and a tree-shrew (Tupaia belangeri) share a unique derived association

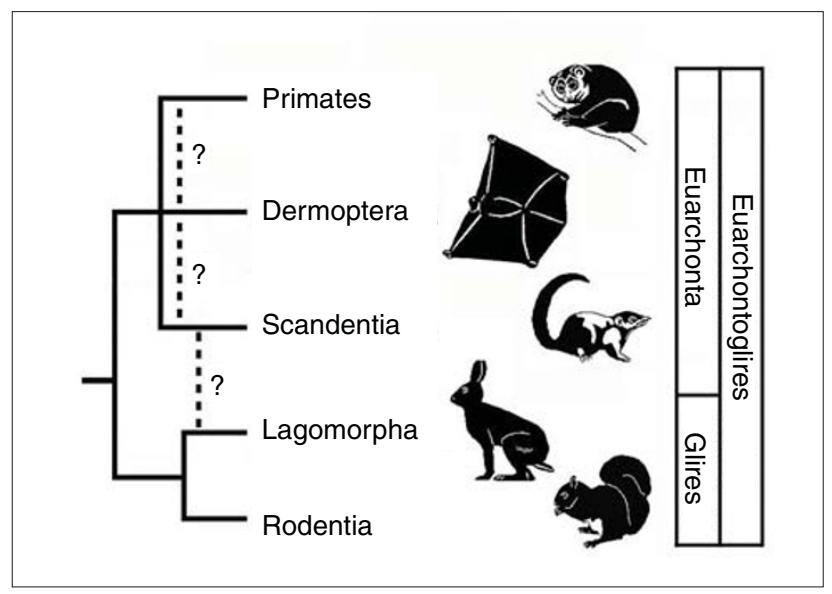

Figure 2

Inferred relationships within the superorder Euarchontoglires. Solid lines indicate branching suggested by a supertree integrating molecular data [20]. Dashed lines with question marks indicate possible alternative links. A basal split between Euarchonta and Glires is often recognized, but some molecular evidence indicates a link between treeshrews (Scandentia) and lagomorphs. Within Euarchonta, colugos (Dermoptera) have been linked either with tree-shrews [8] or with primates [9]. Molecular evidence has generally provided little support for a specific link between tree-shrews and primates.

between two human syntenic segments, an association confirmed by Nie et al. [8] by reverse painting of human chromosomes by $T$. belangeri and $G$. variegatus probes. Moreover, this association is borne on a large autosomal chromosome that is seemingly identical in both. Nie et al. [8] thus provide more evidence for the hypothesis that Scandentia and Dermoptera have a closer phylogenetic relationship to each other than either of them has to Primates. This is confirmation of previous studies indicating that colugos and tree-shrews constitute a monophyletic group [31]. Such a group, labeled Sundatheria, was, for example, indicated by cladistic analysis of dental features [32], and several authors have reported molecular evidence linking colugos to tree-shrews [29,33-36].

But phylogenetic studies are never simple, and another recent study has interpreted DNA sequence and genomic data as showing a closer association of colugos to primates. Janecka et al. [9] combined two independent molecular approaches to explore relationships within Euarchonta: screening of almost 200,000 protein-coding exons to identify rare deletions, and generation of a phylogenetic tree using a 14-kb DNA sequence dataset from nuclear genes. The monophyly of Euarchonta was supported by three specific deletions. No specific deletions linked colugos to tree-shrews. However, seven deletions were common to colugos and primates, whereas tree-shrews and primates 
shared only a single deletion. A relationship between colugos and primates was also indicated by the phylogenetic tree generated from DNA sequences. Overall, the results thus indicate that colugos are closer to primates than to tree-shrews.

So the relationships among colugos, tree-shrews and primates still await resolution (see Figure 2). It is evident, however, that any eventual solution will require convergence at the molecular level, because of the mosaic distribution of shared derived features identified in different studies. Some apparent conflicts may be attributable to polymorphism in common ancestors followed by differential lineage sorting. Despite remaining problems, some provisional conclusions are permissible. First, the superorder Euarchontoglires is uniformly supported by molecular studies, even though no strong morphological evidence favored inclusion of colugos. The prevalent interpretation among morphologists was that bats and colugos are sister groups [6,11], a conclusion resoundingly rejected by all molecular studies. Second, subdivision of Euarchontoglires into two monophyletic sister groups, Euarchonta and Glires, has generally received most support, but there have been several divergent findings. Tree-shrews have sometimes emerged as a basal offshoot in Euarchontoglires or have even been linked specifically to lagomorphs, thus disrupting the monophyly of Glires. Third, a link between colugos and primates within Euarchontoglires has frequently emerged from molecular studies [20], whereas inferred relationships between treeshrews and colugos or primates have been less consistent and far more variable.

A general drawback in many studies has been inclusion of only a single colugo (usually the Philippine rather than the Malayan species). Taking a single representative for an isolated mammalian group can generate misleading results because of long-branch attraction. The study by Janecka et al. [9] laudably included both Cynocephalus and Galeopterus. One immediate benefit of this was demonstration of a surprisingly deep divergence between the two colugos, indicated at approximately 20 million years ago. This not only bolstered the validity of generic separation but also alleviated the effects of long-branch attraction.

Whatever the eventual outcome, colugos must clearly be considered in future discussions of primate evolution. Morphological comparisons between colugos, tree-shrews and primates, rare in the past, are now mandatory. Consideration of colugos will doubtless throw new light on key issues. To take just one example, the brain of colugos is unusually small relative to body size and morphologically very primitive [17]. If colugos are close relatives of primates and/or tree-shrews, this means that any advanced features in tree-shrews and primates are almost certainly convergent, confirming one past interpretation [17]. As both Nie et al. [8] and Janecka et al. [9] noted, proper understanding of morphological and genomic evolution of primates requires identification of the sister group, and colugos (with or without tree-shrews) are definite candidates. For this reason, determination of a draft genome sequence for colugo should certainly be a high priority [37].

\section{References}

I. Simpson GG: The principles of classification and a classification of mammals. Bull Am Mus Nat Hist 1945, 85: I-350.

2. Lim N: Colugo: The Flying Lemur of South-East Asia. Singapore: Draco; 2007.

3. Gregory WK: The orders of mammals. Bull Am Mus Nat Hist 1910, 27:1-524.

4. Butler PM: The skull of Ictops and the classification of the Insectivora. Proc Zool Soc Lond 1956, 126:453-48I.

5. McKenna MC: Toward a phylogenetic classification of the Mammalia. In: Phylogeny of the Primates. Edited by Luckett WP, Szalay FS. New York: Plenum Press; 1975: 21-46.

6. Novacek MJ, Wyss AR: Higher-level relationships of the recent eutherian orders: morphological evidence. Cladistics 1986, 2:257287.

7. McKenna MC, Bell SK: Classification of Mammals Above the Species Level. New York: Columbia University Press; 1997.

8. Nie W, Fu B, O'Brien PCM, Wang J, Su W, Tanomtong A, Volobouev V, Ferguson-Smith MA, Yang F: Flying lemurs - the "flying tree-shrews"? Molecular cytogenetic evidence for a Scandentia-Dermoptera sister clade. BMC Biol 2008, 6:18.

9. Janecka JE, Miller W, Pringle TH, Wiens F, Zitzmann A, Helgen KM, Springer MS, Murphy WJ: Molecular and genomic data identify the closest living relative of primates. Science 2007, 318:792-794.

10. Leche W: Über die Säugethiergattung Galeopithecus: eine morphologische Untersuchung. Kgl Svensk Vetensk-Akad Handl I886, 2I:I-92.

II. Silcox MT, Bloch JI, Sargis EJ, Boyer DM: Euarchonta (Dermoptera, Scandentia, Primates). In The Rise of the Placental Mammals. Edited by Rose KD, Archibald JD. Baltimore: Johns Hopkins University Press; 2005:127-I 44.

12. Martin RD: Primate Origins and Evolution: A Phylogenetic Reconstruction. New Jersey: Princeton University Press; 1990.

13. Beard KC: Gliding behaviour and palaeoecology of the alleged primate family Paromomyidae (Mammalia, Dermoptera). Nature 1990, 345:340-341.

14. Beard KC: Phylogenetic systematics of the Primatomorpha, with special reference to Dermoptera. In Mammal Phylogeny. Volume 2: Placentals. Edited by Szalay FS, Novacek MJ, McKenna MC. New York: Springer-Verlag; I 993: I 29- I 50.

15. Kay RF, Thorington RW, Houde P: Eocene plesiadapiform shows affinities with flying lemurs not primates. Nature 1990, 345:342344.

16. Kay RF, Thewissen JGM, Yoder AD: Cranial anatomy of Ignacius graybullianus and the affinities of the Plesiadapiformes. Amer J Phys Anthropol 1992, 89:477-498.

17. Martin RD: Primate origins: plugging the gaps. Nature 1993, 363: 223-234.

18. Novacek MJ: Mammalian phylogeny: Shaking the tree. Nature 1992, 356:121-125.

19. Murphy WJ, Eizirik E, O'Brien SJ, Madsen O, Scally M, Douady CJ, Teeling E, Ryder OA, Stanhope MJ, de Jong WW, Springer MS: Resolution of the early placental mammal radiation using Bayesian phylogenetics. Science 200I, 294:2348-235I.

20. Bininda-Emonds ORP, Cardillo M, Jones KE, MacPhee RDE, Beck RMD, Grenyer R, Price SA, Vos RA, Gittleman JL, Purvis A: The delayed rise of present-day mammals. Nature 2007, 446:507-5I 2 .

21. Waddell PJ, Ota R: A phylogenetic foundation for comparative mammalian genomics. Genome Inform 200 I, 12:14I-154. 
22. Kriegs JO, Churakov G, Kiefmann M, Jordan U, Brosius J, Schmitz $\mathrm{J}$ : Retroposed elements as archives for the evolutionary history of placental mammals. PLoS Biol 2006, 4:e9l.

23. Kriegs JO, Churakov G, Jurka J, Brosius J, Schmitz J: Evolutionary history of 7SL RNA-derived SINEs in Supraprimates. Trends Genet 2007, 23:|58-|6|.

24. Bailey WJ, Slightom JL, Goodman M: Rejection of the "flying primate" hypothesis by phylogenetic evidence from the $\varepsilon$-globin gene. Science 1992, 256:86-89.

25. Porter CA, Goodman M, Stanhope MJ: Evidence on mammalian phylogeny from sequences of exon 28 of the von Willebrand factor gene. Mol Phylogenet Evol 1996, 5:89-101.

26. Schmitz J, Ohme M, Zischler H: The complete mitochondrial genome of Tupaia belangeri and the phylogenetic affiliation of Scandentia to other eutherian orders. Mol Biol Evol 2000, 17:13341343

27. Hudelot C, Gowri-Shankar V, Jow H, Rattray M, Higgs PG: RNAbased phylogenetic methods: application to mammalian RNA sequences. Mol Phylogenet Evol 2003, 28:24I-252.

28. Arnason U, Adegoke JA, Bodin K, Born EW, Esa YB, Gullberg A, Nilsson MA, Short RV, Xu XF, Janke A: Mammalian mitogenomic relationships and the root of the eutherian tree. Proc Natl Acad Sci USA 2002, 99:8I5I-8I56.

29. Murphy WJ, Eizirik E, Johnson WE, Zhang YP, Ryder OA, O'Brien SJ: Molecular phylogenetics and the origins of placental mammals. Nature 200I, 409:6|4-6I8.

30. Schmitz J, Ohme M, Suryobroto B, Zischler H: The colugo (Cynocephalus variegatus, Dermoptera): The primates' gliding sister? Mol Biol Evol 2002, 19:2308-23 I2

31. Sargis EJ: New views on tree shrews: the role of tupaiids in primate supraordinal relationships. Evol Anthropol 2004, 13:56-66.

32. Marivaux L, Bocat L, Chaimanee Y, Jaeger J-J, Marandat B, Srisuk P, Tafforeau P, Yamee C, Welcomme J-L: Cynocephalid dermopterans from the Palaeogene of South Asia (Thailand, Myanmar and Pakistan): systematic, evolutionary and palaeobiogeographic implications. Zool Scripta 2006, 35:395-420.

33. Adkins RM, Honeycutt RL: Molecular phylogeny of the superorder Archonta. Proc Natl Acad Sci USA I99I, 88:10317-1032I.

34. Liu FGR, Miyamoto MM, Freire NP, Ong PQ, Tennant MR, Young TS, Gugel KF: Molecular and morphological supertrees for eutherian (placental) mammals. Science 2001, 291:1786-1789.

35. Springer MS, Murphy W], Eizirik E, O’Brien SJ: Placental mammal diversification and the Cretaceous-Tertiary boundary. Proc Natl Acad Sci USA 2003, 100:1056-106I.

36. Springer MS, Stanhope MJ, Madsen O, de Jong WW: Molecules consolidate the placental mammal tree. Trends Ecol Evol 2004, 19:430-438.

37. Pennisi E: Genomicists tackle the primate tree. Science 2007 , 316:2|8-22I. 